\title{
Introducción a la Sección Especial de Revista Bosque. Avances de una red de sitios de estudios ecológicos a largo plazo en el suroeste de Sudamérica
}

\author{
Introduction to the Special Section of Journal Bosque. \\ Progress towards a long-term ecological studies network in southwestern South America
}

\author{
Juan J Armesto ${ }^{\text {a,b }}$, Mariela Núñez-Ávila ${ }^{\text {a,c* }}$, Pablo Donoso d, Ricardo Rozzi a,e, f \\ a Instituto de Ecología y Biodiversidad, Las Palmeras 3425, Ñuñoa, Santiago, Chile. \\ ${ }^{\mathrm{b}}$ Pontificia Universidad Católica de Chile, Alameda 340, Santiago, Chile. \\ *Autor de correspondencia: ' Universidad de Concepción, Facultad de Ciencias Forestales, Departamento Manejo de Bosques y \\ Medio Ambiente, Victoria 631, Concepción, Chile, tel.: (56)(41)2661437,mnuneza@udec.cl \\ ${ }^{\mathrm{d}}$ Universidad Austral de Chile, Facultad de Ciencias Forestales y Recursos Naturales, \\ Instituto de Bosques y Sociedad, Isla Teja s/n, Valdivia, Chile. \\ e Universidad de Magallanes, Parque Etnobotánico Omora, Puerto Williams, Chile. \\ ${ }^{\mathrm{f}}$ University of North Texas, Department of Philosophy and Religion Studies, Denton, Texas, USA.
}

\section{SUMMARY}

In this foreword to the special section on long-term ecological research in Chile we briefly introduce the main topics and ideas of each of the articles and comment on their relevance in the context of research and monitoring of Chilean ecosystems and in the perspective of global change research.

Key words: ecosystem research, long-term monitoring, global change.

\section{RESUMEN}

En este prefacio a la sección especial, dedicada a la investigación ecológica de largo plazo en Chile, se comentan brevemente los temas tratados en cada uno de los artículos y se destaca su relevancia en el contexto del monitoreo y la investigación sobre los ecosistemas de Chile y en el ámbito del análisis del cambio global.

Palabras clave: investigación en ecosistemas, monitoreo de largo plazo, cambio global.

Una red de sitios de estudios socio-ecológicos a largo plazo u observatorios naturales de largo plazo, que pueda cubrir una parte importante de la diversidad de ecosistemas del planeta, es esencial para entender, predecir y mitigar los impactos de procesos de cambio ambiental (antropogénico y natural) que se desarrollan a través de varias décadas o que presentan una variación temporal alta (Likens y Lindenmayer 2011). Además, a escala regional o de cada país, los sitios de esta red de observatorios naturales pueden proveer líneas de base del estado de los ecosistemas y responder preguntas relevantes para la sustentabilidad y potencial futuro de muchas actividades productivas del país en el largo plazo. Estos sitios, en la medida que son implementados y utilizados como áreas de referencia y experimentación por los investigadores, permiten la realización de estudios comparativos y la evaluación de modelos y teorías ecológicas y socio-ecológicas en un marco que promueve la comunicación entre investigadores y la sociedad, favoreciendo así la valoración y protección de nuestro patrimonio natural (Rozzi et al. 2012). Los artí- culos de esta sección continúan la discusión de aspectos conceptuales, logísticos y de organización de la naciente red de estudios de largo plazo de Chile, diálogo que iniciamos en Punta Arenas el año 2008, y que se materializó en un conjunto de trabajos filosóficos y ecológicos que fueron publicados en un volumen especial de la revista Environmental Ethics, vol. 30 (2008) y de la Revista Chilena de Historia Natural, vol. 83 (2010).

Durante el taller Fortalecimiento de la Red LTSERCHILE realizado en noviembre del 2012 en el Santuario de la Naturaleza Cascada de Las Animas, con apoyos de CONICYT e Iniciativa Científica Milenio, se acordó preparar una sección especial para la revista Bosque (Chile) que revisara las bases conceptuales, investigaciones en curso, y potencial de expansión de la Red Chilena de Sitios de Estudios Socio-Ecológicos a Largo Plazo (Red LTSER- CHILE).

En este prefacio a la sección especial se comentan brevemente los temas tratados en cada uno de los artículos y se destaca su relevancia en el contexto de la investigación socio-ecológica de largo plazo en Chile y en el ámbito del 
análisis de los ecosistemas a escala global, es decir, sus conexiones presentes o potenciales con la red Internacional de Estudios Ecológicos de Largo Plazo (www.ilter.edu), con la cual los autores están formalmente vinculados. La sección incluye siete manuscritos que fueron parte de las presentaciones en el taller citado más arriba, además de introducir algunos manuscritos adicionales que presentan temas prácticos para el desarrollo futuro de la naciente Red LTSER Chile.

El artículo liderado por Manuel Maass, actual presidente de la Red Internacional de Estudios Ecológicos de Largo Plazo (ILTER, International Long-Term Ecological Research Network), describe los estímulos intelectuales y nuevas preguntas que buscan promover que un mayor número de grupos académicos, sobre todo en países en vías de desarrollo, se incorporen a esta red. Esta plataforma de colaboración internacional está motivada por el desarrollo de estudios socioecológicos y transdiciplinarios que ayuden a resolver los complejos desafíos ambientales del siglo 21 .

En el trabajo presentado por Aurora Gaxiola y colaboradores, se presenta una comparación de las investigaciones que se han realizado hasta el momento en los tres sitios fundadores de la Red Chilena de Estudios Socio-Ecológicos de Largo Plazo, vinculados al Instituto de Ecología y Biodiversidad. Los autores identifican que cada sitio ha desarrollado enfoques diferentes que responden a necesidades locales y del interés de los grupos de investigadores que han iniciado y dirigido los estudios ecológicos o socio-ecológicos a largo plazo. También discuten la necesidad de desarrollar enfoques integrados entre los sitios de la Red LTSER-Chile y las de equipamiento y proyecciones de investigación en el contexto de los problemas derivados del cambio global.

Tamara Contador y colaboradores describen la importancia del estudio de largo plazo de los ciclos de vida de los insectos dulce-acuícolas en la Reserva de la Biosfera Cabo de Hornos, Eco-Región Sub-Antártica de Chile, dada sus particularidades en localización, clima y fragilidad frente a los diversos escenarios de cambio climático. Las respuestas de los insectos dulceacuícolas y sus ciclos de vida son muy sensibles a la temperatura, y bajo las condiciones de gradientes altitudinales y latitudinales se han registrado asociaciones entre cambios térmicos y la fenología de los insectos que permiten hacer predicciones bajo distintos escenarios de cambio climático global, fundadas en evidencia empírica que genera una valiosa línea de base de indicadores de cambio en ambientes de alta latitud.

El estudio de Peter Feinsinger describe el ciclo de indagación aplicado, que guía el desarrollo de las investigaciones con un fin de manejo adaptivo y otros estudios comparativos que son centrales a la investigación de largo plazo. Este artículo ofrece herramientas prácticas a los ecólogos aplicados y básicos para que puedan utilizar esta metodo- logía de investigación para plantear, realizar, interpretar y comunicar investigaciones de campo a largo plazo.

Ricardo Rozzi describe su propuesta metodológica para realizar investigaciones en la interfaz entre la filosofía y ecología. Este método de la filosofía ambiental de campo se ha puesto a prueba en las investigaciones realizadas en el Parque Etnobotánico Omora sobre especies emblemáticas, impacto de especies invasoras y propuestas de ecoturismo para el desarrollo local. Se espera que este trabajo transdisciplinario se amplíe a otras áreas de la red LTSER-Chile.

El estudio liderado por Pablo Donoso y Jennifer Zabaleta hace una propuesta de potenciales sitios relevantes para ampliar la cobertura geográfica de la Red LTSER de Chile y mejorar el diagnóstico del estado de los ecosistemas chilenos. Con este fin, evalúan 12 sitios de estudio de largo plazo en términos de su potencial de investigación, educación y vinculación con el medio externo. Proponen la necesidad de establecer criterios para incorporar estos sitios a la red nacional y la necesidad de incluir en la red sitios de eco-regiones aún no representadas.

Mariela Núñez y colaboradores hacen una propuesta conceptual para la consolidación de una red nacional de Estudios Socio-Ecológicos de Largo Plazo en Chile. Para ello, presentan un marco conceptual que puede servir de base a la investigación socio-ecológica, incluyendo preguntas comparativas a ser abordadas de manera transversal, junto a una propuesta concreta de forma de organización, necesidades de infraestructura y costos básicos para la operación de esta red nacional de observatorios naturales.

Se espera que los siete trabajos de esta sección especial contribuyan a motivar a más ecólogos y científicos chilenos a formar parte del gran desafío de fortalecer y expandir las investigaciones ecológicas de largo plazo en el sur de Sudamérica. También se pretende motivar al Estado de Chile para que en su política científica considere el apoyo a los esfuerzos para proyectar en el largo plazo la investigación y el conocimiento de los ecosistemas donde habitamos y desarrollamos nuestra economía, de manera de contribuir a la sustentabilidad social y ambiental.

\section{REFERENCIAS}

Likens G, D Lindenmayer. 2011. A strategic plan for an Australian Long-Term Environmental Monitoring Network. Austral Ecology 36: 1-8.

Rozzi R, J Armesto, J Gutiérrez, F Massardo, GE Likens, CB Anderson, A Poole, K Moses, E Hargrove, A Mansilla, J Kennedy, M Willson, K Jax, C Jones, JB Callicott, M Arroyo. 2012. Integrating ecology and environmental ethics: earth stewardship in the southern end of the Americas. BioScience 62: 226-236. 